\title{
Revisión sistemática sobre la educación ambiental universitaria en Latinoamérica durante la pandemia (2020-2021)
}

\section{Systematic review of university environmental education in Latin America during the pandemic (2020-2021)}

Miguel Sebastián Armesto Céspedes

Universidad César Vallejo, Lima, Perú

marmestoc@ucvvirtual.edu.pe

https://orcid.org/0000-0003-0497-0891

Rony Isaac Vallejos Armas

Universidad Peruana de Ciencias Aplicadas, Lima, Perú

rvallejo@upc.edu.pe

https://orcid.org/0000-0001-8258-1109

Recepción: 19/04/2021 | Aceptación: 15/06/2021 | Publicación: 10/09/2021

\section{Resumen}

En los últimos 50 años, el cuidado del medio ambiente se ha consolidado en la agenda global y regional; y dentro de ella, la educación ambiental (EA) ha sido uno de los pilares, capaz de re direccionar y modificar el comportamiento de los ciudadanos en pro del equilibrio hombrenaturaleza. No obstante, con el desarrollo de la pandemia por COVID-19 y las declaratorias de aislamiento social obligatorio en todas las regiones, se han tenido que replantear las metas programadas. En esta línea, el objetivo del presente trabajo fue sistematizar las experiencias y evidencias respecto a la educación ambiental en las universidades en Latinoamérica durante este contexto. Para ello fueron analizados 11 artículos científicos en español de las siguientes bases de datos: Scopus, Ebsco, Proquest, Scielo y Dialnet. Para dicho análisis, se tuvo en cuenta criterios de inclusión y exclusión; se trabajó con los artículos publicados en español entre el 2020 y 2021. Entre las conclusiones se obtuvo que en Latinoamérica la EA ha sido objeto de investigaciones en los que se priorizó el diagnóstico para sus posteriores planes de mejora e implementación.

Palabras claves: universidad; medio ambiente; conciencia ambiental; COVID-19. 


\begin{abstract}
Over the last 50 years, environmental care has been consolidated in the global and regional agenda; and within it, environmental education (EE) has been one of the mainstays, capable of redirecting and modifying the behavior of citizens in favor of the human-nature balance. However, with the development of the COVID-19 pandemic and the declarations of mandatory social isolation in all regions, the programmed goals have had to be reconsidered. In this line, the objective of the present work was to systematize the experiences and evidence regarding environmental education in universities in Latin America during this context. For this purpose, 11 scientific articles in Spanish from the following databases were analyzed: Scopus, Ebsco, Proquest, Scielo and Dialnet. For this analysis, inclusion and exclusion criteria were taken into account; we worked with articles published in Spanish between 2020 and 2021. Among the conclusions, it was found that in Latin America $\mathrm{AD}$ has been the subject of research in which diagnosis was prioritized for subsequent improvement and implementation plans.
\end{abstract}

Keywords: university; environment; environmental awareness; COVID-19.

\title{
Introducción
}

El presente artículo refleja el análisis de las experiencias en un contexto en el que el COVID- 19 ha impactado en todas las esferas imaginables, obligando al mundo a redefinir sus costumbres, hábitos, formas de interacción y de encarar el proceso educativo (con las limitaciones que esto representa en una sociedad que ha mostrado desiguales maneras de acceder a los recursos). Esta situación ha condicionado y alterado los sectores productivos, en especial en aquellas naciones en las que los indicadores económicos no eran los más altos antes de la pandemia, y donde se podían notar grandes brechas socioeconómicas, las cuales se han evidenciado con lo acontecido (Arriagada, 2020; Tejedor et al., 2020; Cifuentes, 2020), como es el caso de Perú (Delgado, 2020; Llerena y Sánchez, 2020; Jaramillo y Ñopo, 2020)

Los diversos problemas ambientales que han aparecido a lo largo del tiempo, como la desertificación (Silva et al., 2017); los impactos de los gases invernaderos; la gestión ambiental, que en muchos casos no ha sido la idónea por parte de los pobladores, quienes pretenden cubrir sus necesidades básicas en desmedro de su entorno, autoridades (Armesto y Montes de Oca, 2020) y el sector privado (Zenck et al., 2018), el cual suele ampararse en el marco normativo existente en las regiones donde operan para desenvolverse dentro de los límites permisibles, esto con el objetivo de obtener la materia prima necesaria para completar su proceso productivo (Azqueta, 2002). Ante todo, los Estados y autoridades proponen aplicar, en la mayor y mejor medida de lo posible, las regulaciones establecidas para intentar mitigar los impactos en el medio ambiente.

Estos impactos, producidos en el entorno, son productos de la satisfacción de las necesidades poblacionales; y estas, a su vez, debido a los hábitos. En este contexto, la Educación Ambiental (EA), surgida a mediados de los años setenta, aparece con el objetivo de mejorar el cuidado del ambiente, valorando el concepto de calidad de vida (Naciones Unidas, 1975). A pesar de la importancia de esta, en Perú, por ejemplo, el desarrollo de las actividades asociadas obtuvo un respaldo institucional en 2002, con la forma de convenios por parte de las autoridades estatales correspondientes, lo que llevó a aprobar el Plan Operativo Anual en el año 2005. Este plan presentaba un enfoque integral dentro del diseño Curricular Nacional de la Educación Básica 
Regular en el que los materiales elaborados y la implementación de los Proyectos Educativos Institucionales servían como complementos para poder reforzar e interiorizar el proceso en la comunidad educativa.

En este proceso, la EA puede ser entendida como un instrumento capaz de volver responsables a los ciudadanos en cuanto a su entorno, encontrando un equilibrio entre los elementos (Silva et al., 2020); y más aún dentro de la crisis ambiental actual. Ante ello, la intervención en los sistemas educativos se convierten en una estrategia adecuada para revertir a mediano y largo plazo dicha situación (Araya y Muñoz, 2017). Es necesario mencionar que dicho concepto se encuentra asociado al Desarrollo Sostenible, permitiendo que este último tenga más posibilidades de ser alcanzado en función a las adaptaciones y prácticas socioambientales por parte de los diversos actores de la comunidad (Consuegra y Centeno, 2020). Sin embargo, esta debe ser dada de forma transversal, permanente e inclusive dentro de los diversos y variados espacios posibles como en la educación formal, informal y no formal, propiciando valores de respeto, solidaridad, sensibilización (Quintana, 2017; Caro, 2019) y actitud ecológica (Olivos et al., 2013). En síntesis, la EA es el reflejo de la transferencia de aprendizaje en todos los espacios cotidianos con la participación activa de los actores con la intención de consolidar el espíritu eco-responsable de la sociedad en su conjunto (Herrera y Ríos, 2017)

Es necesario tomar en cuenta que el Desarrollo Sostenible (DS) es el resultado de un proceso continuo que está relacionado con la EA (Consuegra y Centeno, 2020; Nay y Cordero, 2019); para ello, se requiere que haya un participación activa por parte de la comunidad educativa y otros actores (Quintero y Solarte, 2019; Jiménez et al., 2018; Rivera et al., 2013; Hernández, 2018); y cuanto más sinergia entre ellos, mejores resultados se obtendrán (Saidón y Claverie, 2016; Del Arco et al., 2021; Silva et al., 2017). La evaluación permanente de los procesos y aprendizajes en el aula le dan consistencia, con lo cual provoca una interiorización de las prácticas ambientales en la vida de los estudiantes (Araya y Muñoz, 2017; Quintana, 2017; Yeşilyurt et al., 2020; Herrera \& Ríos, 2017). Sin embargo, este proceso puede estar condicionado por el entorno del alumno (Olivos et al., 2013). A ello, se suman los impactos de la EA en la conciencia (Vásquez, 2010; Zevallos 2005)

Teniendo en cuenta que la educación es uno de los grandes pilares que pueden impactar estructuralmente en el comportamiento de los ciudadanos (Brantmeier, 2013), la EA promueve una interacción entre el hombre y su entorno bajo diversos elementos como la paz, la justicia y el respeto al ambiente, dentro del diálogo entre los actores (Solís y Barreto, 2020; Sónora y García, 2020; Medir et al., 2016; Consuegra y Centeno, 2020) que se verán reflejadas en un actitud y conciencia responsable frente al entorno (Furtado et al., 2016; Yeşilyurt et al., 2020; Quintero y Solarte, 2019). Dentro de ello cada país de la región ha optado por adaptar la EA a sus sistemas educativos en función a sus características, como en Cuba (Rivera et al., 2013; Santana et al., 2018), Brasil (Furtado et al., 2016; Silva et al., 2016), Chile (Torres et al., 2015; Torres et al., 2017), Colombia (Galvis et al., 2019; Sánchez et al., 2016), Argentina (Saidón y Claverie, 2016), Venezuela (Granados y Moros, 2017) en los que se ha priorizado en el nivel de educación básica o regular. Por ello, en función a lo expuesto, el objetivo del presente artículo ha sido: sistematizar las experiencias y evidencias de la EA en el entorno universitario en Latinoamérica durante la pandemia por COVID-19. Sobre este tema se plantearon dos objetivos específicos. El primero fue 
analizar las estrategias y enfoques metodológicos de las investigaciones seleccionadas. El segundo fue analizar los resultados obtenidos en los artículos revisados.

\section{Metodología}

En la presente investigación, se realizó una revisión sistemática que tuvo como unidad de análisis artículos originales, los cuales permitieron sintetizar la información científica (Ferreira et al., 2011); para ello, es necesario dejar claro el protocolo y el método planificado para dicha revisión (Moher et al., 2016). Dicho análisis se llevó a cabo sobre la "educación ambiental" a partir de los artículos publicados en el año 2020 y 2021 en las siguientes bases de datos: Ebsco, Dialnet, Scielo, Proquest y Scopus. El total de artículos encontrados fue de 570; de ellos, se descartaron aquellos no contaban con los criterios de inclusión: estudios aplicados en universidades, estudios desarrollados en Latinoamérica, investigaciones aplicadas, investigaciones publicadas en español y artículos de acceso abierto. En cuanto a los criterios de exclusión se tomaron en cuenta los siguientes: estudios desarrollados en la educación básica (escuelas), investigaciones teóricas y artículos de discusión, investigaciones aplicadas no desarrolladas en/por universidades e investigaciones aplicadas fuera del contexto latinoamericano. Como resultado, de acuerdo con el método Prisma (Moher et al., 2016), se obtuvo un total de 11 artículos, los cuales cumplen con los objetivos propuestos y constituyen la base para la presente revisión.

\section{Figura 1}

Proceso de revisión PRISMA

\begin{tabular}{|c|c|}
\hline $\begin{array}{l}\text { Índice de } \\
\text { Base de } \\
\text { datos }\end{array}$ & $\begin{array}{l}\text { Artículos registrados en base de datos } \\
\text { (Ebsco, Pro Quest, Scielo y Dialnet) } \\
\text { referidos a Educación ambiental entre el } \\
2020 \text { y } 2021 . \mathbf{n}=\mathbf{5 7 0}\end{array}$ \\
\hline $\begin{array}{l}\text { Índice por } \\
\text { nivel de } \\
\text { educación }\end{array}$ & $\begin{array}{l}\text { De los } 570 \text { artículos, quedaron } 25 \text { por } \\
\text { encontrarse en el nivel de educación superior }\end{array}$ \\
\hline $\begin{array}{l}\text { Índice por } \\
\text { región de } \\
\text { análisis } \\
\end{array}$ & $\begin{array}{l}\text { De los } 25 \text { artículos, } 18 \text { fueron los } \\
\text { seleccionados dentro de Latinoamérica }\end{array}$ \\
\hline $\begin{array}{l}\text { Índice por } \\
\text { objetivos }\end{array}$ & Estudios incluidos $\mathbf{n}=\mathbf{1 1}$ \\
\hline
\end{tabular}

Nota: se detalla el proceso de depuración de las referencias en las bases de datos siguiendo el modelo PRISMA 
Tabla 1

Resultado de selección en los repositorios

\begin{tabular}{|c|c|c|c|c|c|c|c|c|c|}
\hline Repositorio & Código & $\begin{array}{l}\text { Combinación } \\
\text { de búsqueda }\end{array}$ & Resultados & $\begin{array}{l}\begin{array}{l}\text { Temática } \\
\text { (nivel }\end{array} \\
\text { superior } \\
\text { universitario) }\end{array}$ & Tipo & Región & Acceso & Objetivos & $\begin{array}{l}\text { Artículos } \\
\text { seleccionados }\end{array}$ \\
\hline EBSCO & A1 & $\begin{array}{l}\text { "Educación } \\
\text { ambiental" }\end{array}$ & 199 & 12 & 4 & 11 & 11 & 11 & 4 \\
\hline PROQUEST & B1 & $\begin{array}{l}\text { "Educación } \\
\text { ambiental" }\end{array}$ & 20 & 0 & 1 & 0 & 0 & 0 & 0 \\
\hline SCOPUS & $\mathrm{C} 1$ & $\begin{array}{l}\text { "Educación } \\
\text { ambiental" }\end{array}$ & 13 & 0 & 3 & 0 & 0 & 0 & 0 \\
\hline SCIELO & D1 & $\begin{array}{l}\text { "Educación } \\
\text { ambiental" }\end{array}$ & 50 & 2 & 0 & 2 & 2 & 2 & 2 \\
\hline DIALNET & E1 & $\begin{array}{l}\text { "Educación } \\
\text { ambiental" }\end{array}$ & 288 & 11 & 6 & 5 & 5 & 5 & 5 \\
\hline Total & & & 570 & 25 & 14 & 18 & 18 & 18 & 11 \\
\hline
\end{tabular}

En la tabla 1 se puede apreciar el proceso de selección; donde, en función a los repositorios elegidos y el motor de búsqueda (que fue "educación ambiental") se pudieron obtener 570 artículos. Sin embargo, al tomar en cuenta el nivel de educación superior, la región (Latinoamérica), y su vinculación con los objetivos se seleccionaron solo 11 investigaciones.

\section{Resultados}

En función a los objetivos formulados, se expondrán los resultados de las investigaciones de los 11 artículos seleccionados.

\section{Características de los artículos}

De acuerdo con el proceso de revisión Prisma, podemos observar que de las once (11) fuentes seleccionadas en la presente revisión, la mayoría de trabajos publicados en Latinoamérica, durante el contexto de la pandemia por COVID-19, se encuentran en Colombia y México, regiones desde las cuales se han realizado tres (3) investigaciones en cada una sobre la EA; le sigue Cuba con dos (2) trabajos publicados; y, finalmente se encuentran Perú, Ecuador y Honduras, países en los que se ha desarrollado una investigación en cada región.

\section{Tabla 2}

Artículos incluidos en la revisión

\begin{tabular}{lll}
\hline Repositorio & Autor, año & País \\
\hline Scielo & (Miranda et al., 2020) & México \\
Scielo & (Solís y Barreto, 2020) & Colombia \\
Ebsco & (Méndez-Cadena et al., 2020) & México
\end{tabular}




\begin{tabular}{lll} 
Repositorio & Autor, año & País \\
\hline Ebsco & (Pedraza, 2020) & Colombia \\
Ebsco & (Suazo y Torres, 2021) & Honduras \\
Ebsco & (Gil et al., 2020) & Colombia \\
Dialnet & (Santacruz et al., 2021) & Perú \\
Dialnet & (Cantú, 2020) & México \\
Dialnet & (Camacho y Valdés, 2020) & Ecuador \\
Dialnet & (Almeida et al., 2020) & Cuba \\
Dialnet & (Márquez et al., 2020) & Cuba \\
\hline
\end{tabular}

\section{Figura 2}

Porcentaje de fuentes de acuerdo con la región de procedencia

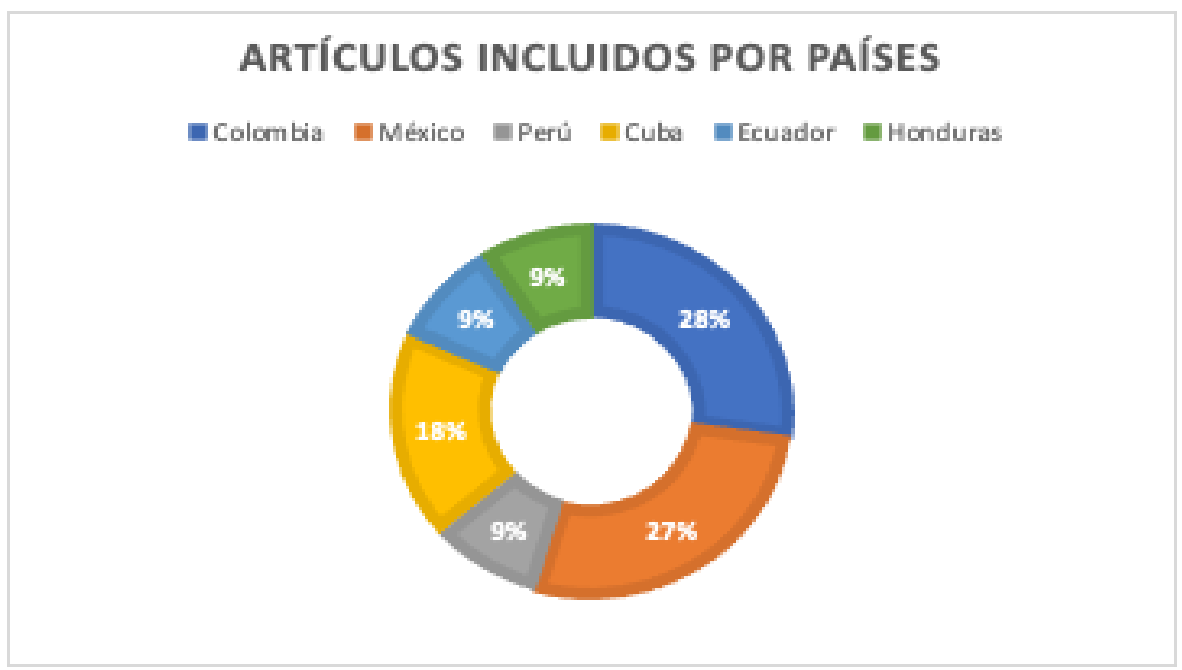

Nota: se detalla el origen de los artículos por países

\section{Estrategias y metodología empleada}

La revisión de las investigaciones nos revela que no existe una preferencia respecto al enfoque metodológico. Podemos observar que, de acuerdo a lo declarado por los autores, no existe una metodología que sobresalga respecto de las otras. Apreciamos que cuatro (4) de las investigaciones optaron por un enfoque cuantitativo y cuatro (4) también por un enfoque cualitativo. Solo tres (3) de las investigaciones se desarrollaron con una metodología mixta.

Por otro lado, sobre el instrumento para el recojo de información, podemos apreciar que la mayoría de las investigaciones, siete (7) de ellas, eligieron el cuestionario. De estas, cuatro (4) fueron aplicadas a estudiantes, dos (2) a docentes y una (1) a estudiantes y docentes. Del mismo 
modo, podemos notar que cuatro (4) investigaciones optaron por instrumentos distintos como cuestionario-entrevista, entrevista, análisis documental y grupos focales-entrevistas.

\section{Tabla 3}

Estrategias y enfoques metodológicos

\begin{tabular}{lll}
\multicolumn{1}{c}{ Autor, año } & \multicolumn{1}{c}{ Metodología } & \multicolumn{1}{c}{ Instrumento de recolección de datos } \\
\hline (Miranda et al., 2020) & Enfoque mixto & Cuestionario y entrevista \\
(Solís y Barreto, 2020) & Cuantitativo & Cuestionario (estudiantes) \\
(Méndez-Cadena et al., 2020) & Cualitativo & Cuestionario (estudiantes) \\
(Pedraza, 2020) & Cualitativo & Grupos focales y entrevistas (docentes, \\
(Suazo y Torres, 2021) & Cuantitativo & estudiantes, administrativos) \\
(Gil et al., 2020) & Mixta & Cuestionario (docentes) \\
(Santacruz et al., 2021) & Mixta & Cuestionario (estudiantes) \\
(Cantú, 2020) & Cuantitativo & Cuestionario (docentes) \\
(Camacho y Valdés, 2020) & Cuantitativo & Cuestionario (estudiantes) \\
(Almeida et al., 2020) & Cualitativo & Cuestionario (docentes y estudiantes) \\
(Márquez et al., 2020) & Cualitativa & Entrevistas (docentes y estudiantes) \\
\hline
\end{tabular}

\section{Figura 3}

\section{Enfoque metodológico e instrumentos utilizados}

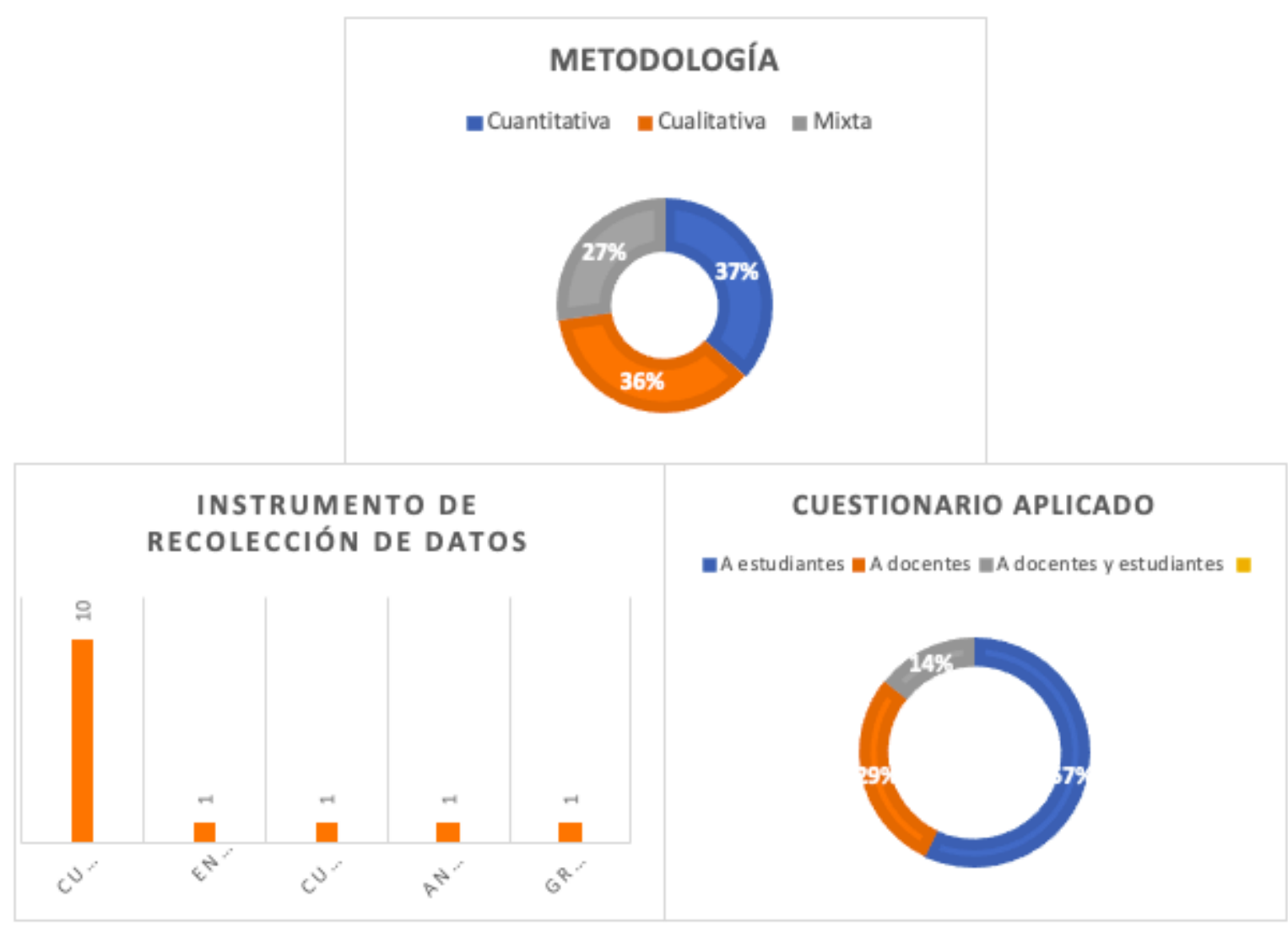


Nota: se detalla en la primera figura la metodología utilizada en las investigaciones analizadas, posteriormente, se detallan los instrumentos aplicados.

En la figura 3 puede apreciarse la preferencia de aplicar cuestionarios al momento de recoger los datos, y a ello se puede complementar que la unidad de análisis se orienta a los estudiantes; es decir, se recurre a la percepción, experiencia y opinión de uno de los actores del proceso en la educación ambiental, y en este caso es quien la recibe. En un segundo momento es orientado al punto de vista del docente y por último se aplican los instrumentos en ambos.

\section{Conclusiones y objetivos de los artículos revisados}

En el contexto de pandemia por el COVID-19, de las once investigaciones analizadas, hemos podido realizar una clasificación en cuatro objetivos claros. La mayoría plantearon objetivos descriptivos. El primero de estos podríamos decir que fueron guiados por el hecho de evaluar en función de la percepción de los estudiantes (Solís y Barreto, 2020), la percepción y el conocimiento de los estudiantes (Suazo y Torres, 2021) y los aportes de una institución en el cumplimiento de los objetivos del desarrollo sostenible (Márquez et al., 2020). Un segundo grupo tuvo como meta identificar para implementar y elaborar una propuesta (Miranda et al., 2020; Pedraza, 2020), diseñar una estrategia para tener una cultura ambiental (Almeida et al., 2020), para conocer la actitud y describir el razonamiento de los estudiantes (Cantú, 2020; Gil et al., 2020). En un tercer grupo encontramos que el objetivo fue analizar en función de la incidencia de la malla curricular en la formación de la educación ambiental y el nivel de competencia que los estudiantes tienen sobre el tema (Méndez-Cadena et al., 2020; Camacho y Valdés, 2020). Finalmente, el objetivo de una investigación fue el de interpretar la relación que existe entre el liderazgo que ejercen los docentes y el desarrollo sostenible verde, que implica una educación ambiental por parte de la comunidad universitaria (Santacruz, 2021).

\section{Tabla 4}

\section{Conclusiones obtenidas}

\begin{tabular}{|c|c|c|c|}
\hline Repositorio & $\begin{array}{l}\text { Autor, } \\
\text { año }\end{array}$ & Objetivos & Conclusiones \\
\hline Scielo & $\begin{array}{l}\text { (Miranda } \\
\text { et al., } \\
2020 \text { ) }\end{array}$ & $\begin{array}{l}\text { Identificar la dimensión ambiental } \\
\text { en el plan de estudio de una } \\
\text { escuela para luego implementar un } \\
\text { taller dirigido a conservar } \\
\text { manglares }\end{array}$ & $\begin{array}{l}\text { Se hace presente la necesidad de incluir la } \\
\text { dimensión ambiental en el currículo de forma } \\
\text { transformacional, debido a los resultados } \\
\text { positivos respecto a la conservación de } \\
\text { manglares }\end{array}$ \\
\hline Scielo & $\begin{array}{l}\text { (Solís y } \\
\text { Barreto, } \\
2020)\end{array}$ & $\begin{array}{l}\text { Evaluar la percepción de los } \\
\text { estudiantes sobre la sostenibilidad } \\
\text { dentro de un marco específico (la } \\
\text { Cátedra de la Paz colombiana) }\end{array}$ & $\begin{array}{l}\text { Es necesario enfocar la sostenibilidad desde } \\
\text { un punto de vista holístico e integral. }\end{array}$ \\
\hline Ebsco & $\begin{array}{l}\text { (Méndez- } \\
\text { Cadena et } \\
\text { al., 2020) }\end{array}$ & $\begin{array}{l}\text { Analizar la incidencia que tiene la } \\
\text { formación alrededor de los cursos } \\
\text { sobre la mitigación al cambio } \\
\text { climático en la representación } \\
\text { social }\end{array}$ & $\begin{array}{l}\text { La representación del CC está asociado a la } \\
\text { temperatura en aumento, a ello se añade la } \\
\text { reducida relación entre los efectos de la } \\
\text { conceptualización en las acciones de } \\
\text { mitigación }\end{array}$ \\
\hline
\end{tabular}




\begin{tabular}{|c|c|c|c|}
\hline Repositorio & $\begin{array}{c}\text { Autor, } \\
\text { año }\end{array}$ & Objetivos & Conclusiones \\
\hline Ebsco & $\begin{array}{l}\text { (Pedraza, } \\
\text { 2020) }\end{array}$ & $\begin{array}{l}\text { Desarrollar en función a las } \\
\text { experiencias de la comunidad } \\
\text { educativa una propuesta sobre la } \\
\text { gestión ambiental dentro del } \\
\text { currículo }\end{array}$ & $\begin{array}{l}\text { Se evidenciaron limitaciones a nivel } \\
\text { conceptual respecto al medio ambiente y su } \\
\text { vinculación son el currículo dentro de un } \\
\text { marco discursivo claro, dificultando la gestión } \\
\text { de esta }\end{array}$ \\
\hline Ebsco & $\begin{array}{l}\text { (Suazo y } \\
\text { Torres, } \\
\text { 2021) }\end{array}$ & $\begin{array}{l}\text { Evaluar las percepciones, } \\
\text { conocimiento y enseñanza sobre el } \\
\text { Cambio Climático y los riesgos } \\
\text { que producen los desastres }\end{array}$ & $\begin{array}{l}\text { Los resultados remarcan la necesidad de } \\
\text { fortalecer conocimientos sobre riesgos y sobre } \\
\text { investigación aplicada para ser desarrolladas } \\
\text { en las facultades respectivas }\end{array}$ \\
\hline Ebsco & $\begin{array}{l}\text { (Gil et al., } \\
2020 \text { ) }\end{array}$ & $\begin{array}{l}\text { Describir el razonamiento de los } \\
\text { estudiantes de biología, ciencias } \\
\text { naturales y educación ambiental } \\
\text { sobre los ecosistemas }\end{array}$ & $\begin{array}{l}\text { Existe una comprensión sobre el } \\
\text { funcionamiento de los ecosistemas, sin } \\
\text { embargo, dependiendo de la carrera la } \\
\text { posición tenía variaciones }\end{array}$ \\
\hline Dialnet & $\begin{array}{l}\text { (Santacruz } \\
\text { et al., } \\
\text { 2021) }\end{array}$ & $\begin{array}{l}\text { Interpretar la relación entre el } \\
\text { liderazgo transformacional y el } \\
\text { desarrollo sostenible verde por } \\
\text { parte de los docentes de la UNHV } \\
\text { (Huánuco) }\end{array}$ & $\begin{array}{l}\text { Existe una relación directa y significativa } \\
\text { entre el liderazgo transformacional ejercido } \\
\text { por los docentes y el desarrollo sostenible } \\
\text { verde, tanto en alumnos como en el área } \\
\text { administrativa. }\end{array}$ \\
\hline Dialnet & $\begin{array}{l}\text { (Cantú, } \\
\text { 2020) }\end{array}$ & $\begin{array}{l}\text { Conocer la actitud de los } \\
\text { estudiantes respecto al medio } \\
\text { ambiente. }\end{array}$ & $\begin{array}{l}\text { Existe una actitud positiva y participativa en } \\
\text { favor del cuidado del medio ambiente en los } \\
\text { estudiantes universitarios que previamente } \\
\text { llevaron un curso de ecología y biodiversidad. }\end{array}$ \\
\hline Dialnet & $\begin{array}{l}\text { (Camacho } \\
\text { y Valdés, } \\
\text { 2020) }\end{array}$ & $\begin{array}{l}\text { Analizar y determinar el nivel de } \\
\text { competencia ambiental de los } \\
\text { estudiantes de la especialidad de } \\
\text { ingeniería civil de } 10 \\
\text { universidades en Ecuador. }\end{array}$ & $\begin{array}{l}\text { El nivel de competencia ambiental de los } \\
\text { estudiantes es muy bajo en temas ambientales. } \\
\text { Esto debido a que no existen materias en la } \\
\text { facultad que les imparten conocimientos en } \\
\text { estos temas ( } 3 \text { universidades) o porque la } \\
\text { materia que se imparte sobre medio ambiente } \\
\text { no es transversal en la facultad ( } 7 \\
\text { universidades). }\end{array}$ \\
\hline Dialnet & $\begin{array}{l}\text { (Almeida } \\
\text { et al., } \\
\text { 2020) }\end{array}$ & $\begin{array}{l}\text { Diseñar una estrategia para la } \\
\text { formación de una cultura } \\
\text { ambiental en los estudiantes de } \\
\text { Derecho }\end{array}$ & $\begin{array}{l}\text { Hubo una respuesta y actitud favorable hacia } \\
\text { los temas medioambientales en los estudiantes } \\
\text { de Derecho. }\end{array}$ \\
\hline Dialnet & $\begin{array}{l}\text { (Márquez } \\
\text { et al., } \\
\text { 2020) }\end{array}$ & $\begin{array}{l}\text { Evaluar los aportes del Centro de } \\
\text { Estudios de Medio } \\
\text { Ambiente y Recursos Naturales, de } \\
\text { la } \\
\text { Universidad de Pinar del Río } \\
\text { "Hermanos } \\
\text { Saíz Montes de Oca", en los } \\
\text { Objetivos de Desarrollo } \\
\text { Sostenible. }\end{array}$ & $\begin{array}{l}\text { Los resultados fueron positivos y de mucho } \\
\text { aporte en cuanto a investigaciones sobre } \\
\text { medio ambiente y educación ambiental. Se } \\
\text { obtuvo una buena respuesta validada en las } \\
\text { tesis, artículos y ponencias en las que } \\
\text { participaron los estudiantes de dicha } \\
\text { universidad. }\end{array}$ \\
\hline
\end{tabular}




\section{Figura 4}

\section{Objetivos desarrollados en los artículos}

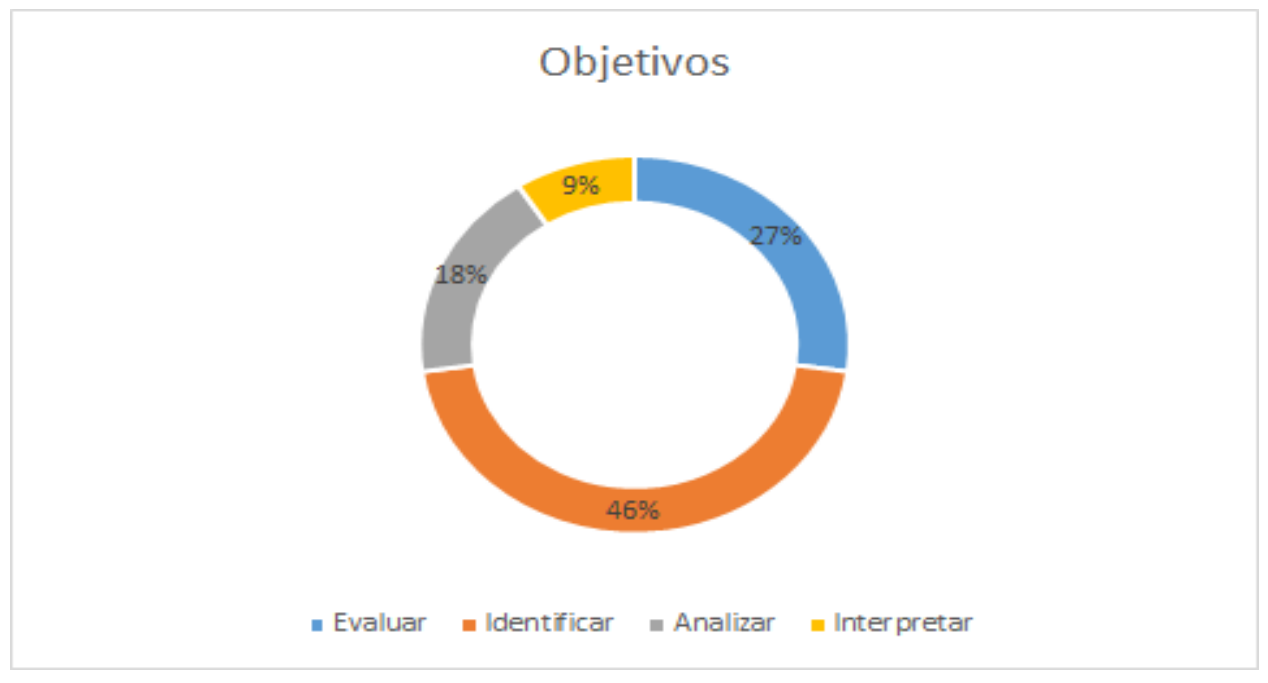

Nota: se detallan los porcentajes de los objetivos (en común) entre los artículos analizados

La figura 4 muestra claramente cómo las investigaciones analizadas priorizan la identificación, tomándolo como una etapa prioritaria para luego proponer soluciones o mejoras al proceso descrito. Ello debido a que, en este contexto, las dinámicas han cambiado y urge identificar situaciones, errores, fallas o aciertos con el objetivo de continuar con la práctica educativa.

\section{Conclusiones}

El análisis realizado, evidencia que Colombia y México son los países con la mayor cantidad de investigaciones en español en Latinoamérica alrededor de la EA en las universidades durante la pandemia (entre marzo 2020 y marzo 2021). Respecto a los enfoques de investigación empleados, se aprecia un equilibrio en la elección y desarrollo de los mismos: enfoques mixtos (3), cuantitativos y cualitativos (4).

Por otro lado, de los objetivos planteados en los artículos revisados, podemos concluir en la importancia que los investigadores le han otorgado a la identificación y evaluación para construir propuestas que permitan mejorar y desarrollar estrategias y métodos de concientización ambiental en los estudiantes universitarios. Entre ambas metas, podemos apreciar que constituyen el $73 \%$ de los objetivos trabajados, lo cual es un indicio de la importancia de la EA en el nivel superior de la educación.

Dentro del contexto pandémico en que nos encontramos, la EA como parte de un proceso integral capaz de crear conciencia en los ciudadanos respecto a su entorno, se hace aún más necesaria teniendo en cuenta los impactos que están siendo generados por la COVID-19. Dicho 
esto, el proceso no puede detenerse y las universidades deben adecuarse a las condiciones actuales para seguir con ello.

Es importante resaltar la poca producción científica que se ha publicado en lengua castellana, desde Latinoamérica, sobre la materia en cuestión. Lo cual hace un llamado hacia la comunidad científica para abordar un tema que compete a toda la población y el bienestar de la misma. Es necesario desarrollar estrategias educativas que permitan la formación del individuo y se reconozca como parte de un ecosistema que debe proteger y respetar.

\section{Referencias bibliográficas}

Almeida, M., Ledea, B. y Galafell, A. (2020). Cultura ambiental del profesional de la carrera de Derecho en Educación Superior. Koinonia, 5 (1), 893-910. http://doi.org/10.35381/r.k.v5i1.977

Araya, D. y Muñoz, D. (2017). Educación ambiental y cultura evaluativa. Algunas reflexiones para la construcción de eco-consciencias. Estudios Pedagógicos, 43(1), 389-403. https://doi.org/10.4067/S0718-07052017000100022

Arriagada, P. (2020). Pandemia Covid-19: educación a distancia. O las distancias en la educación. Revista Internacional de Educación Para La Justicia Social, 9(3). https://revistas.uam.es/riejs/article/download/12396/12222/32897

Armesto, M. y Montes de Oca, H. (2020). La gestión del talento humano en la sostenibilidad ambiental: revisión sistemática de artículos científicos disponibles en Acceso abierto en español. Anuario Facultad de Ciencias Económicas y Administrativas. 2. 57-67. https://anuarioeco.uo.edu.cu

Azqueta, D. (2002). Introducción a la economía ambiental. Madrid: McGraw-Hill.

Brantmeier, E. (2013). Toward a critical peace education for sustainability. Journal of peace education, 10(3), 242-258. https://doi.org/10.1080/17400201.2013.862920

Camacho, M. y Valdés, M. (2020). Análisis del grado de desarrollo de la competencia ambiental en estudiantes de Ingeniería Civil de la Universidad Católica de Santiago de Guayaquil. $\begin{array}{llll}\text { Entrevista Académica, } & 2 & \text { 38-55. }\end{array}$ https://dialnet.unirioja.es/servlet/articulo?codigo=7806106

Cantú, P. (2020). Actitudes proambientales en jóvenes universitarios. Ciencia y Educación, 4(2), 67-74. https://doi.org/10.22206/cyed.2020.v4i2.pp67-74

Caro, M. (2019). Recuperación y protagonismo del cuerpo en la educación ambiental. Revista Internacional de Filosofía y Teoría Social, 87, 127-137. https://produccioncientificaluz.org/index.php/utopia/article/view/27561

Cifuentes, J. (2020). Consecuencias en los Niños del Cierre de Escuelas por Covid-19: El Papel del Gobierno, Profesores y Padres Javier. Revista Internacional de Educación Para La Justicia Social, 9. https://revistas.uam.es/riejs/article/view/12216

Consuegra, G. y Centeno, M. (2020). Acercamiento a la conceptualización de la educación ambiental para el desarrollo sostenible. Discussing the Conceptualization of Environmental Education for Sustainable Development. Revista Cubana de Educación Superior, 39 (2), 22. http://scielo.sld.cu/pdf/rces/v39n2/0257-4314-rces-39-02-e18.pdf

Del Arco, I., Silva, P. \& Flores, O. (2021). University teaching in times of confinement: The light and shadows of compulsory online learning. Sustainability (Switzerland), 13(1), 1-16. 
https://doi.org/10.3390/su13010375

Delgado, D. (2020). La COVID-19 en el Perú: una pequeña tecnocracia enfrentándose a las consecuencias de la desigualdad. Análisis Carolina, 1-16. https://doi.org/10.33960/ac_26.2020

Ferreira, I., Urrútia, G., y Alonso, P. (2011). Revisiones sistemáticas y metaanálisis: bases conceptuales e interpretación. Revista Española de Cardiología, 64(8), 688-696. https://doi.org/10.1016/j.recesp.2011.03.029

Furtado, N., Furtado, P., Coelho, F. y Andrade, A. (2016). Prácticas De Educación Ambiental Y Sostenibilidad Aplicada a la formación ciudadana. Geográfica Académica, 10 (2), 30-40. http://eds.b.ebscohost.com/eds/pdfviewer/pdfviewer?vid=0\&sid=197c1b14-2a64-457a85bf-e8c3e61c8951\%40sessionmgr120

Galvis, C., Perales, F. y Ladino, Y. (2019). ¿Cómo implementan la Educación Ambiental en sus aulas profesores de centros educativos rurales colombianos? Contextos Educativos. Revista de Educación, 23(23), 101. https://doi.org/10.18172/con.3519

Gil, J., Navarro, C. y Pinzón, I. (2020). Razonamiento funcional y causal sobre el desequilibrio ecosistémico en estudiantes universitarios. Educación y Humanismo, 22(39), 1-22. https://doi.org/10.17081/eduhum.22.39.4081

Granados, B. y Moros, J. (2017). Gestión estratégica del docente en la enseñanza de la educación ambiental en el nivel de educación primaria. International Journal of Good Conscience, 12(3), 78-100. https://doi.org/10.18601/01207555.n23.05

Hernández, D. (2018). Curricular strategy of environmental education for the students of the career degree in Education. Pedagogy-psychology. Revista Luna Azul, 46(46), 369-386. https://doi.org/10.17151/luaz.2018.46.19

Herrera, D. y Ríos, D. (2017). Educación ambiental y cultura evaluativa. Algunas reflexiones para la construcción de eco-consciencias. Estudios Pedagógicos (Valdivia), 1(43), 389-403. https://www.redalyc.org/pdf/1735/173553246022.pdf

Jaramillo, M. y Ñopo, H. (2020). El impacto del COVID-19 sobre la economía peruana. Economía UNAM, $17(51)$,

136-146. www.latinamerica.undp.org/content/rblac/en/home/library/crisis_prevention_and_recover y/covid-

Llerena, R. y Sánchez, C. (2020). Emergencia, gestión, vulnerabilidad y respuestas frente al impacto de la pandemia COVID-19 en el Perú. SciELO, 16. https://preprints.scielo.org/index.php/scielo/preprint/view/94/129

Márquez, D., Linares, M., Hernández, R. y Márquez, L. (2020). Implementación de los Objetivos del Desarrollo Sostenible desde un Centro de Estudios Universitario. Mendive. Revista de Educación, $18(2)$ 336-346. http://mendive.upr.edu.cu/index.php/MendiveUPR/article/view/1799

Medir, H., Heras, R. y Magin, C. (2016). Una propuesta evaluativa para actividades de educación ambiental para la sostenibilidad. Educación XX1, 19(1), 331-356. https://doi.org/10.5944/educXX1.14226

Méndez-Cadena, M., Crispín, A., Vargas, A. y Ruiz, P. (2020). De la representación social del cambio climático a la acción el caso de estudiantes universitarios. Revista Mexicana de Investigación Educativa, $\quad$ 25(87), 1043-1068. http://www.scielo.org.mx/scielo.php?script=sci_arttext\&pid=S1405$66662020000401043 \& \operatorname{lng}=$ es\&tlng=es.

Miranda, A., Bedolla, R., Bedolla, J. y Sánchez, O. (2020). Educación sustentable no formal para 
conservar los manglares en zonas costeras con estudiantes de Sociología, UAGro. In RIDE Revista Iberoamericana para la Investigación y el Desarrollo Educativo 10(20). https://doi.org/10.23913/ride.v10i20.618

Moher, D., Shamseer, L., Clarke, M., Ghersi, D., Liberati, A., Petticrew, M., Shekelle, P., Stewart, L. \& Group, P. (2016). Preferred reporting items for systematic review and meta-analysis protocols (PRISMA-P) 2015 statement. Revista Española de Nutrición Humana y Dietética, 20(2), 148-160. https://doi.org/10.1186/2046-4053-4-1

Nay, M. y Cordero, M. (2019). Educación Ambiental y Educación para la Sostenibilidad: historia, $\begin{array}{lllll}\text { fundamentos } \mathrm{y} \text { tendencias. Revista } & \text { 17(02). }\end{array}$ https://doi.org/10.15665/encuent.v17i02.661

Olivos, P., Aragonés, J., y Navarro, O. (2013). Educación ambiental: itinerario ambientales y conducta con conectividad, preocupaciones en la naturaleza y su relación Environmental. Revista Latinoamericana de Psicología, 45(3), 501-511. https://doi.org/10.14349/rlp.v45i3.1490

Pedraza, Y. (2020). La investigación-acción-participación para problematizar la ambientalización curricular universitaria. Tecné Episteme y Didaxis: TED, 47, 93-109. https://doi.org/10.17227/ted.num47-9516

Pineda, C., López, X., Wehncke, E. y Maldonado, B. (2018). Construir sociedades comprometidas con el entorno natural: educación ambiental en niños del sur de Morelos, México. Región y Sociedad, 30(72). https://doi.org/10.22198/rys.2018.72.a896

Quintana, R. (2017). La educación ambiental y su importancia en la relación sustentable: HombreNaturaleza-Territorio. Revista Latinoamericana de Ciencias Sociales, Niñez y Juventud, 15, 927-949. https://doi.org/10.11600/1692715x.1520929042016

Quintero, M. y Solarte, M. (2019). Las concepciones de ambiente inciden en el modelo de enseñanza de la educación ambiental. Ciencias Sociales, 15(2), 130-147. https://doi.org/10.18041/1900-3803/entramado.2.5602

Rivera, M., Hernández, B. y Castillo, G. (2013). Programa de educación ambiental en el Consejo Popular Capitán San Luis. Revist CientíficA Avances, 15(2), 201-210. https://www.mendeley.com/viewer/?fileId=52b00e29-ae39-0dbd-1cf9d63b32b5d573\&documentId=1d9443c7-2517-3791-bdd1-707ba4a50b95

Saidón, M. y Claverie, J. (2016). Percepciones de docentes y directores sobre los factores que promueven u obstaculizan la educación ambiental en escuelas del Área Metropolitana de Buenos Aires. Ciência \& Educação (Bauru), 22(4), 993-1012. https://doi.org/10.1590/1516-731320160040010

Sánchez, V., Gómez, C., Coronado, C. y Valenzuela, W. (2016). Imaginarios ambientales y de educación ambiental de los estudiantes y docentes de la Institución Educativa Agrotécnico Mixto, municipio de Belén de los Andaquíes (Caquetá). Revista Universidad Simón Bolivar, 19(32), 126-144. http://doi.org/10.17081/eduhum.19.32.2537

Santacruz, A., Montenegro, H., Pizarro, A. y Estacio, H. (2021). Liderazgo transformacional y desarrollo sostenible ambiental verde en docentes de la Universidad Nacional Hermilio Valdizán. Revista de Comunicación de La SEECI, 53, 135-151. https://doi.org/10.15198/seeci.2020.53.135-151

Santana, E., Bocourt, J. y Casas, M. (2018). La educación ambiental comunitaria para los niños y niñas de la comunidad Soroa. Revista Científica Avances, 20(2), 122-132. http://www.ciget.pinar.cu/ojs/index.php/publicaciones/article/view/334/1227

Silva, A., Nascimento, V., Farias, J., Andrade, A. y Silva, E. (2016). Sustentabilidade E Educação 
Ambiental: a (Re) Construção Da Consciência Ecológica Nas Escolas Públicas De Fortaleza-. ACTA Geográfica, 87-102. http://doi.org/10.5654/acta.v0i0.3758

Silva, A., Canganjo, A. y López, J. (2020). Procedimientos metodológicos para desarrollar el derecho y la educación ambiental en los estudiantes universitarios de la carrera Ingeniería de los Recursos Hídricos. Opuntia Brava, 12(4), 226-235. http://opuntiabrava.ult.edu.cu/index.php/opuntiabrava/article/view/1135

Silva, M., Barros, A. y Gomes, B. (2017). Desertificación y conocimiento escolar en el Sérido Potiguar: la educación ambiental como posibilidad de formación y ciudadanía. Geosaberes, 8(16), 41. https://doi.org/10.26895/geosaberes.v8i16.606

Solís, C. y Barreto, C. (2020). La visión de la educación ambiental de estudiantes de maestría en pedagogía en el marco de la Cátedra de la Paz en Colombia. Formación Universitaria, 13(2), 153-166. https://doi.org/10.4067/S0718-50062020000200153

Sónora, L. y García, A. (2020). Climántica: un proyecto pedagógico-social y de educación ambiental en la lucha contra el cambio climático. Pedagogía Social: Revista Interuniversitaria, 26, 203-228. https://doi.org/10.7179/PSRI_2020.36.04

Suazo, L. y Torres, A. (2021). Percepciones, conocimiento y enseñanza de cambio climático y riesgo de desastres en universidades hondureñas. Formación Universitaria, 14(1), 225236. https://doi.org/10.4067/s0718-50062021000100225

Tejedor, S. Cervi, L., Tusa, F. y Parola, A. (2020). Educación en tiempos de pandemia: reflexiones de alumnos y profesores sobre la enseñanza virtual universitaria en España, Italia y Ecuador. Revista Latina de Comunicación Social, 78, 1-21. https://doi.org/10.4185/rlcs2020-1466

Torres, L., Benavides, J., Latoja, C. y Novoa, E. (2017). Presencia de una Educación Ambiental basada en conocimiento, actitudes y prácticas en la enseñanza de las ciencias naturales en establecimientos municipales de la ciudad de Los ángeles, Chile. Estudios Pedagógicos, 43(3), 311-323. https://doi.org/10.4067/S0718-07052017000300018

Torres, L., Mesina, N., Salmanca, B. y Sepúlveda, C. (2015). Efectos de la enseñanza interdisciplinaria en la educación ambiental sobre los conocimientos, valores y actitudes ambientales de estudiantes de segundo ciclo básico (Los Ángeles, Región de Biobío, Chile). Revista Complutense de Educación, 27(3), 1139-1155. https://doi.org/10.5209/rev_RCED.2016.v27.n3.47551

Yeşilyurt, M., Balakoğlu, M. y Erol, M. (2020). El Impacto de las Actividades de Educación Ambiental en la Conciencia Ambiental y las Expresiones Visuales de los Estudiantes de Primaria. Qualitative Research in Education, 9(2), 188-216. https://doi.org/10.17583/qre.2020.5115

Zenck, M., Urrutia, P. y Ríos, I. (2018). El desarrollo sostenible como política pública Caso Gobierno Autónomo Descentralizado de Quito. Regions and Cohesion, 8(3), 45-69. https://doi.org/10.3167/reco.2018.080304 